\title{
Penetration of magnetosonic waves into the magnetosphere: influence of a transition layer
}

\author{
A. S. Leonovich ${ }^{1,3}$, V. V. Mishin ${ }^{1,3}$, and J. B. Cao ${ }^{2,3}$ \\ ${ }^{1}$ Institute of Solar-Terrestrial Physics (ISTP), Russian Academy of Science, Siberian Branch, Irkutsk 33, P.O.Box 4026, \\ 664033, Russia \\ ${ }^{2}$ Center for Space Science and Applied Research, Chinese Academy of Science, China \\ ${ }^{3}$ Russian-Chinese Joint Research Center on Space Weather, China
}

Received: 22 January 2002 - Revised: 29 October 2002 - Accepted: 12 November 2002

\begin{abstract}
We have constructed a theory for the penetration of magnetosonic waves from the solar wind into the magnetosphere through a transition layer in a plane-stratified model for the medium. In this model the boundary layer is treated as a region, inside of which the parameters of the medium vary from values characteristic for the magnetosphere, to values typical of the solar wind. It is shown that if such a layer has sufficiently sharp boundaries, then magnetosonic eigenoscillations can be excited inside of it. The boundaries of such a layer are partially permeable for magnetosonic waves. Therefore, if the eigen-oscillations are not sustained by an external source, they will be attenuated, because some of the energy is carried away by the oscillations that penetrate the solar wind and the magnetosphere. It is shown that about $40 \%$ of the energy flux of the waves incident on the transition layer in the magnetotail region penetrate to the magnetosphere's interior. This energy flux suffices to sustain the stationary convection of magnetospheric plasma. The total energy input to the magnetosphere during a time interval of the order of the substorm growth phase time is comparable with the energetics of an average substorm.
\end{abstract}

Key words. Magnetospheric physics (MHD waves and instabilities; solar wind-magnetosphere interactions) - Space plasma physics (kinetic and MHD theory)

\section{Introduction}

The assumption that the solar wind MHD oscillations can play a significant role in the energetics of the Earth's magnetosphere was made by Axford (1964). The cited paper was followed by a number of theoretical publications devoted to the study of the penetration of the MHD waves from the solar wind into the magnetosphere. Noteworthy is a paper by McKenzie (1970), who constructed a theory for the penetration of magnetosonic waves through the magnetopause, treated as a tangential discontinuity of parameters of the me-

Correspondence to: A. S. Leonovich (leon@iszf.irk.ru) dium. Subsequently, Verzariu (1973) and Wolfe and Kaufmann (1975) performed an integration of reflection and transmission coefficients of magnetosonic waves over the spectrum of solar wind oscillation frequencies. These integral coefficients were used to estimate the energy flux transported by magnetosonic waves from the solar wind to the magnetosphere. Based on those estimates it was found that no more than $1-2 \%$ of the solar wind energy flux penetrates to the magnetosphere's interior and the resulting flux through the dayside magnetosphere is $10^{9} \mathrm{~W}$. This value is an order of magnitude smaller than required for sustaining a stationary magnetospheric convection. Therefore, following the publication of the cited references, nobody was earnest about the contribution of the solar wind magnetosonic waves to the energetics of magnetospheric processes. Mishin (1996) showed, however, that upstream waves with a large amplitude can play a vital part in the dynamics of collisionless magnetospheric plasma.

In the cited papers, a direct calculation of the energy fluxes of incident reflected and transmitted waves was not carried out. For such a calculation, it is necessary to specify the spectrum of solar wind oscillations, both for frequencies and for wave-vectors of incident waves. Relatively ample observational evidence on solar wind oscillation spectra was secured during the past two decades. This has enabled us to perform, in our first paper (Leonovich and Mishin 1999), a complete calculation of the energy flux of magnetosonic waves transferred to the magnetosphere. In this paper we have shown that about $40 \%$ of incident wave energy flux penetrates into the magnetosphere. This provides enough energy to drive magnetospheric convection. Verzaiu (1973) and Wolfe and Kaufmann (1975) considered the transfer of magnetosonic oscillation energy through the front part of the magnetosphere, where the solar wind flows around it in the subsonic mode. Unlike the cited references, we investigated the penetration of magnetosonic waves through the tail part of the magnetosphere, where the solar wind flows around it in the supersonic mode. Herewith, the spectral region of magnetosonic oscillations that are able to penetrate deep into the 
magnetosphere is considerably extended.

However, all of the cited references used the model of the medium in which the magnetosphere and the solar wind are separated by a common sharp boundary. Papers by McKenzie (1970), Verzariu (1973), Wolfe and Kaufmann (1975), Leonovich and Mishin (1999) used a model with one sharp boundary as well. In fact, between the magnetosphere and the solar wind there is a relatively wide boundary layer. There is the magnetosheath, the region between the magnetopause and the bow shock. In addition, under the magnetopause there is also a boundary layer which, in its morphological characteristics, is divided into the low-latitude boundary layer (LLBL) and the high-latitude boundary layer (HLBL). In this paper we consider the problem of the propagation of fast magnetosonic waves from the solar wind to the magnetosphere through a boundary layer. The model of the medium used for this purpose corresponds to a greater extent to the structure of the nightside HLBL in the tail part of the magnetosphere. We hope, however, that results obtained in this study can also be used in a qualitative analysis of the process of penetration of magnetosonic waves in some regions of the dayside part of the magnetosphere at a relatively large distance from the subsolar point.

For the sake of simplicity, it will be assumed that the boundary layer has two sharp boundaries. Such a structure of the boundary layer is in fact observed from time to time in the magnetosphere (Akasofu et al., 1973; Skopke and Paschmann, 1978). A similar model of the boundary layer was used in analyses of the Kelvin-Helmholtz instability at the magnetopause (Yumoto and Saito, 1980; Lee et al., 1981; Farrugia et al., 2000) and of the structure of magnetosound waveguide eigen-oscillations in the outer magnetosphere (Mann et al., 1999).

It should be noted that most often the inner boundary of the boundary layer is more diffuse when compared with the outer boundary. The structure of the MHD oscillation field in the boundary layer with a smoothly varying structure was analyzed in detail by De Keyser et al. (1999), De Keyser and Gadez (2001), Rezeau and Belmont (2001), and Belmont and Rezeau (2001). It was shown that these oscillations can trigger the field line reconnection process at the magnetopause (Rezeau and Belmont, 2001; Belmont and Rezeau, 2001). The amplitude of such Alfvén oscillations, with the typical parameters of the moderately disturbed magnetosphere, can exceed the amplitude of magnetosonic waves that excite them (Leonovich, 2001a). However, the presence of these resonance Alfvén oscillations has a relatively small influence on the structure of magnetosonic waves. The reason is that only a small part of the energy flux of magnetosonic waves goes into the excitation of Alfvén oscillations. Consequently, when investigating the transfer process of the energy flux of magnetosonic waves through the magnetopause, the presence of Alfvén resonances in the transition layer can be neglected. In this paper we use the model of the medium in which such a resonance interaction is absent.

In this paper we consider the process of wave energy transport from the solar wind to the magnetosphere by fast mag- netosonic waves. The model of the boundary layer with two sharp boundaries used in this study leads to a somewhat larger (compared to models of the medium with a smooth transition layer, Leonovich, 2001b) reflection coefficient of magnetosonic waves from the boundary layer to the solar wind. For that reason, our values of the energy flux of these waves from the solar wind to the magnetosphere can be regarded as a lower bound. Furthermore, when integrating the energy fluxes over the spectrum of magnetosonic waves incident on the magnetosphere from the solar wind, we allowed for the presence of "transparency windows" in regions of variations of their frequency and wave vector. The "transparency window" are such ranges of variation of frequencies and wave vectors, that in each of the layers considered here the magnetosonic waves can propagate freely in the direction of a normal to their boundaries.

This paper is organized as follows; in Sect. 2, the model of the medium is described, and a matching of solutions of the MHD equations representing magnetosonic waves in the solar wind, the transition layer and the magnetosphere is performed. Eigenmodes of magnetosonic oscillations of the transition layer are investigated in Sect. 3. In Sect. 4, we investigate the dispersion equation of magnetosonic waves and determine the ranges of frequency and wave-vector variation which form "transparency windows" for these waves in the solar wind and in the magnetosphere. Section 5 presents the expressions for the energy flux of magnetosonic waves that are amenable to a numerical integration over oscillation spectra. In Sect. 6, a numerical integration of energy fluxes over the spectrum of waves incident on the transition layer is performed using a model spectral function, and the results obtained are discussed. The main results of this study are summarized in the Conclusions.

\section{Model of the medium, and matching of solutions of the MHD equations}

We now choose a plane-stratified model of the medium (Fig. 1) that assumes a transition layer of a thickness $\Delta$ (region II in Fig. 1), separating the solar wind (region I) and the magnetosphere (region III). The assumption that layer II has two sharp boundaries implies that both its thickness $\Delta$ and the wavelength of the oscillations under consideration, in the direction across the layer, must be much larger than the gyroradius of plasma ions $\rho_{i}$. As will be evident from subsequent calculations, these conditions are satisfied for the oscillations that we address here. The Cartesian system $(x, y, z)$ will be chosen such that the axis $z$ is directed normal to the transition layer boundaries $(z=0$ and $z=\Delta)$ into the magnetosphere, the axis $x$ is directed along the geomagnetic field $B^{\mathrm{III}}$, and the axis $y$ completes a right-handed coordinate system. In the designation of parameters of the medium the upper index $i=$ I,II,III indicates the region number in accordance with Fig. 1.

Plasma and magnetic field parameters will be considered to be homogeneous in each of regions I-III and to change 


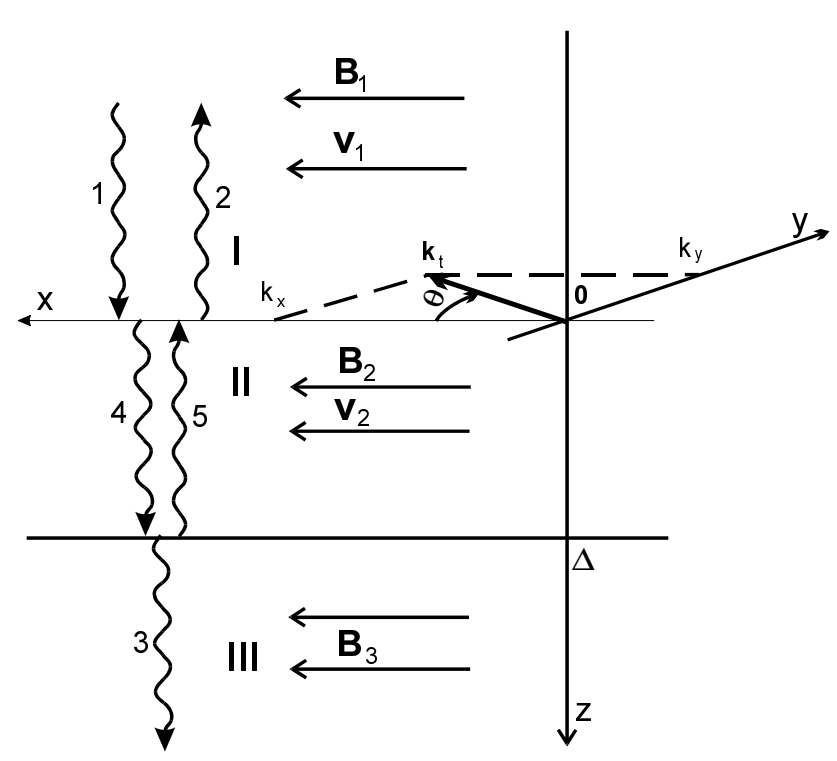

Fig. 1. Model of the medium and coordinate system. Roman numerals number the regions: I - solar wind, II - transition layer, III - magnetosphere. Arabic numerals number the waves: 1 - incident on the transition layer, 2 - reflected from the transition layer, 3 transmitted to the magnetosphere, 4 - transmitted to the transition layer's interior, 5 - reflected inside the transition layer.

their values abruptly in passing through the boundaries $z=0$ and $z=\Delta$. For the sake of simplicity assume that the velocity vector $\boldsymbol{v}^{i}$ and the magnetic field vector $\boldsymbol{B}^{i}(i=\mathrm{I}, \mathrm{II}, \mathrm{III})$ are directed along the axis $x$. Of course, this restricts the validity of our model of the medium to the region of the high-latitude boundary layer, which, however, occupies the main part of the surface of the nightside magnetosphere. Within the magnetosphere $(i=\mathrm{III})$ the plasma is at rest $v^{\mathrm{III}}=0$, and in regions I and II it moves with the velocities $v^{\mathrm{I}}$ and $v^{\mathrm{II}}$, so that $v^{\mathrm{I}}>v^{\mathrm{II}}$. The plasma dynamics in regions I-III will be described in an ideal MHD approximation. At steady state the law of conservation of total pressure follows from the MHD equations:

$P+\frac{B^{2}}{8 \pi}=$ const,

where $P$ is the gas kinetic pressure of the plasma, and $B^{2} / 8 \pi$ is magnetic pressure.

Taking into consideration the homogeneity of undisturbed parameters of the medium, we shall seek the solutions of the initial system of MHD equations linearized with respect to the disturbance in the form of a Fourier harmonic

$\varphi=\tilde{\varphi} \exp \left[-\mathrm{i} \omega t+\mathrm{i} \mathbf{k}_{t} \mathbf{r}_{t}+\mathrm{i} k_{z} z\right]$,

where $\tilde{\varphi}$ is the amplitude of any one of the disturbed parameters, $\omega$ is the oscillation frequency, and $\mathbf{k}_{t}=\left(k_{x}, k_{y}\right)$ and $k_{z}$ are, respectively, the tangential and normal components of the wave vector (see Fig. 1). Three branches of MHD oscillations exist in each of the layers under consideration (see Anderson, 1963): Alfvén waves with the dispersion law $\bar{\omega}^{(i)^{2}}=k_{x}^{2} A^{(i)^{2}}, \mathrm{i}=\mathrm{I}$,II,III , where $A^{(i)}=B^{(i)} / \sqrt{4 \pi \rho^{(i)}}$ is the Alfvén velocity in layer $i$, fast and slow magnetosonic waves with the dispersion law

$\bar{\omega}^{(i)^{4}}-\bar{\omega}^{(i)^{2}} k^{2}\left(A^{(i)^{2}}+S^{(i)^{2}}\right)+k^{2} k_{x}^{2} A^{(i)^{2}} S^{(i)^{2}}=0$,

where $\bar{\omega}^{(i)}=\omega-k_{x} v^{(i)}$ is the oscillation frequency with the inclusion of the Doppler shift in a medium traveling with the velocity $v^{(i)}, k^{2}=k_{x}^{2}+k_{y}^{2}+k_{z}^{2}$, and $S^{(i)}=\sqrt{\gamma P^{(i)} / \rho^{(i)}}$ is the sound velocity in layer $i$. Alfvén waves, according to their dispersion law, propagate strictly along the magnetic field $\boldsymbol{B}$ (i.e. in our model of the medium - along the axis $x$ ) and hence do not participate in the energy transfer along the axis $z$. In what follows, therefore, we will be examining only magnetosonic waves which can propagate along the axis $z$.

Consider the problem of the transmission of a magnetosonic wave from the solar wind to the magnetosphere through the transition layer. This problem, according to its statement, is quite analogous to the problem of the transmission of MHD waves from the magnetosphere to the ground through the ionosphere (see Greifinger, 1972; Leonovich and Mazur, 1991). From the solar wind the magnetosonic wave is incident on the transition layer (index 1 in Fig. 1) which is partially reflected into the solar wind (index 2) and partially penetrates into the transition layer (index 4). The wave that has penetrated the transition layer, in turn, penetrates partially to the magnetosphere's interior (index 3) and is partially reflected from the boundary $z=0$ (index 5). When the wave is incident on each of the boundaries, these boundaries change their shape depending on the structure of the incident and reflected waves. The matching conditions on each of the disturbed boundaries have the form (see McKenzie, 1970)

$\left\{\tilde{P}+\frac{B^{(i)} \tilde{B}_{x}}{4 \pi}\right\}=0$,
$\left\{\tilde{B}_{z}+B^{(i)} \frac{\partial \tilde{\xi}_{z}}{\partial x}\right\}=0$,

$\left\{\frac{\mathrm{d} \tilde{\xi}_{z}}{\mathrm{~d} t}-\tilde{v}_{z}\right\}=0$

where the braces identify an abrupt change in the enclosed quantities when passing through the disturbed boundary. Here, $\tilde{P}$ is the disturbed gas kinetic pressure of the plasma, $\tilde{B}_{x}$ is the longitudinal (along the undisturbed magnetic field $\boldsymbol{B})$ component of the oscillation magnetic field, $\tilde{\xi}_{z}$ is the normal component of plasma displacement in the wave, and $\tilde{v}_{z}$ is the normal component of the disturbed velocity. Equations (3a) and (3b) follow directly from the linearized MHD equations when they are integrated across the disturbed boundary. Equation (3c) is defined by the assumption that, in passing through the boundary (tangential discontinuity), the tangential component of the wave vector is conserved, i.e. the oscillation of the boundary may be represented as

$\left.\tilde{\xi}_{z}\right|_{z=0, \Delta}=\eta \exp \left(\mathbf{i} \mathbf{k}_{t} \mathbf{r}_{t}-\mathrm{i} \omega t\right)$ 
where $\eta$ is the oscillation amplitude of the boundary. Using Eq. (4) and eliminating from Eqs. (3) the oscillation amplitude of the boundary $\eta$, we obtain the following matching conditions on the boundary $z=0$

$$
\begin{aligned}
& \bar{\omega}^{\mathrm{II}}\left(\tilde{v}_{z 1}+\tilde{v}_{z 2}\right)=\bar{\omega}^{\mathrm{I}}\left(\tilde{v}_{z 4}+\tilde{v}_{z 5}\right), \\
& B^{\mathrm{II}}\left(\tilde{B}_{z 1}+\tilde{B}_{z 2}\right)=B^{\mathrm{I}}\left(\tilde{B}_{z 4}+\tilde{B}_{z 5}\right), \\
& \tilde{P}_{1}+\tilde{P}_{2}+B^{\mathrm{I}} \frac{\tilde{B}_{x 1}+\tilde{B}_{x 2}}{4 \pi}=\tilde{P}_{4}+\tilde{P}_{5}+B^{\mathrm{II}} \frac{\tilde{B}_{x 4}+\tilde{B}_{x 5}}{4 \pi},
\end{aligned}
$$

and on the boundary $z=\Delta$

$$
\begin{aligned}
& \bar{\omega}^{\mathrm{III}}\left(\tilde{v}_{z 4} \mathrm{e}^{\mathrm{i} k_{z}^{\mathrm{II}} \Delta}+\tilde{v}_{z 5} \mathrm{e}^{-\mathrm{i} k_{z}^{\mathrm{II}} \Delta}\right)=\bar{\omega}^{\mathrm{II}} \tilde{v}_{z 3} \mathrm{e}^{\mathrm{i} k_{z}^{\mathrm{III}} \Delta}, \\
& B^{\mathrm{III}}\left(\tilde{B}_{z 4} \mathrm{e}^{\mathrm{i} k_{z}^{\mathrm{II}} \Delta}+\tilde{B}_{z 5} \mathrm{e}^{-\mathrm{i} k_{z}^{\mathrm{II}} \Delta}\right)=B^{\mathrm{II}} \tilde{B}_{z 3} \mathrm{e}^{\mathrm{i} k{ }_{z}^{\mathrm{III}} \Delta}, \\
& \left(\tilde{P}_{4}+B^{\mathrm{II}} \frac{\tilde{B}_{x 4}}{4 \pi}\right) \mathrm{e}^{\mathrm{i} k_{z}^{\mathrm{II}} \Delta}+\left(\tilde{P}_{5}+B^{\mathrm{II}} \frac{\tilde{B}_{x 5}}{4 \pi}\right) \mathrm{e}^{-\mathrm{i} k_{z}^{\mathrm{II}} \Delta} \\
& =\left(\tilde{P}_{3}+B^{\mathrm{III}} \frac{\tilde{B}_{x 3}}{4 \pi}\right) \mathrm{e}^{\mathrm{i} k_{z}^{\mathrm{III}} \Delta} .
\end{aligned}
$$

Here, subscripts $1-5$ number the wave parameters, in accordance with the wave indices in Fig. 1. In Eqs. $(5 d-5 f)$, the component of the wave vector $k_{z}^{(i)}$ now has the superscript index $\mathrm{i}$, corresponding to the layer number $\mathrm{i}=\mathrm{I}$-III, because at the specified $\omega$ and $\boldsymbol{k}_{t}$, one may express $k_{z}$ from the dispersion Eq. (2)

$k_{z}^{(i)^{2}}=-k_{t}^{2}+\frac{\bar{\omega}^{(i)^{4}}}{\left(\bar{\omega}^{(i)^{2}}-k_{x}^{2} c_{s}^{(i)^{2}}\right) a_{s}^{(i)^{2}}}$,

where the designations $a_{s}^{(i)}=\sqrt{A^{(i)^{2}}+S^{(i)^{2}}}$ and $c_{s}^{(i)}=$ $A^{(i)} S^{(i)} / a_{s}^{(i)}$ are introduced. Deciding between the signs of $k_{z}^{(i)}$ in Eqs. (5d-5f) is determined by the condition $k_{z}^{(i)}>0$. From the disturbed equations of ideal MHD, we have (see McKenzie, 1970)

$k_{z}^{(i)}\left(P^{(i)}+B^{(i)} \frac{\tilde{B}_{x}}{4 \pi}\right)=\frac{\bar{\omega}^{(i)^{2}}-k_{x}^{2} A^{(i)^{2}}}{\bar{\omega}^{(i)}} \rho^{(i)} v_{z}^{(i)}$.

Substitution of this relationship into (5) transforms Eqs. (5a), $(5 \mathrm{c}),(5 \mathrm{~d})$ and $(5 \mathrm{f})$ to a system of equations closed with respect to $\tilde{v}_{z}$. It can give the relationships

$\tilde{v}_{z 2}=R \tilde{v}_{z 1,} \quad \tilde{v}_{z 3}=T \tilde{v}_{z 1}$

where it is designated

$R=\frac{\left(1-Z_{1} Z_{2}\right) \cos k_{z}^{\mathrm{II}} \Delta+\mathrm{i}\left(Z_{1}-Z_{2}\right) \sin k_{z}^{\mathrm{II}} \Delta}{\left(1+Z_{1} Z_{2}\right) \cos k_{z}^{\mathrm{II}} \Delta-\mathrm{i}\left(Z_{1}+Z_{2}\right) \sin k_{z}^{\mathrm{II}} \Delta}$,

$T=\frac{\bar{\omega}^{\mathrm{III}}}{\bar{\omega}^{\mathrm{I}}} \frac{2 \exp \left(-\mathrm{i} k_{z}^{\mathrm{III}} \Delta\right)}{\left(1+Z_{1} Z_{2}\right) \cos k_{z}^{\mathrm{II}} \Delta-\mathrm{i}\left(Z_{1}+Z_{2}\right) \sin k_{z}^{\mathrm{II}} \Delta}$,
$Z_{1}=\frac{\rho^{\mathrm{II}}}{\rho^{\mathrm{I}}} \frac{k_{z}^{\mathrm{I}}}{k_{z}^{\mathrm{II}}} \frac{\bar{\omega}^{\mathrm{II}^{2}}-k_{x}^{2} A^{\mathrm{II}^{2}}}{\bar{\omega}^{\mathrm{I}^{2}}-k_{x}^{2} A^{\mathrm{I}^{2}}}$,

$Z_{2}=\frac{\rho^{\mathrm{III}}}{\rho^{\mathrm{II}}} \frac{k_{z}^{\mathrm{II}}}{k_{z}^{\mathrm{III}}} \frac{\bar{\omega}^{\mathrm{III}}-k_{x}^{2} A^{\mathrm{III}}}{\bar{\omega}^{\mathrm{II}}-k_{x}^{2} A^{\mathrm{II}^{2}}}$.

Here, $R$ and $T$, according to the system of notation used by McKenzie (1970) and Verzariu (1973), are said to be the reflection and transmission coefficients of the wave. In the limit $\Delta=0$ the expressions (8) and (9) go over into corresponding expressions in the absence of the transition layer (McKenzie, 1970). When $\boldsymbol{v}^{(i)}=0$ and $\boldsymbol{B}^{(i)}=0$, the expressions (8) and (9) give the reflection and transmission coefficients of sound through a plane transition layer (see Brekhovskikh, 1980).

One can see that the coefficients $R$ and $T$ turn out to be modulated with a change in the frequency $\omega$ and the tangential wave vector $\boldsymbol{k}_{t}$. This is attributable to the presence of magnetosonic eigen-oscillations of the transition layer.

\section{Eigenmodes of the transition layer}

The dispersion equation for the eigenmodes of the transition layer may be obtained by modifying the originally formulated problem in such a way that no wave incident on the transition layer from the solar wind is present. This can also be achieved through a limiting transition $R \rightarrow \infty$ and $T \rightarrow \infty$, i.e. by making the denominator in the expressions (8) and (9) vanish. The dispersion equation for eigenmodes has the form

$\tan k_{z}^{\mathrm{II}} \Delta=-\mathrm{i} \frac{\left(1+Z_{1} Z_{2}\right)}{\left(Z_{1}+Z_{2}\right)} \equiv-\mathrm{i} Z$.

A general solution of this equation can be found numerically. This does not come within the province of the problem formulated in this paper, however. For the purposes of illustration we will therefore limit ourselves to a simple particular case $\boldsymbol{k}_{t}=0$, when the solution of Eq. (10) can be obtained analytically. In this case $Z_{1}=\left(\rho^{\mathrm{II}} / \rho^{\mathrm{I}}\right)\left(a_{s}^{\mathrm{II}} / a_{s}^{\mathrm{I}}\right)$ and $Z_{2}=\left(\rho^{\mathrm{III}} / \rho^{\mathrm{II}}\right)\left(a_{s}^{\mathrm{III}} / a_{s}^{\mathrm{II}}\right)$ are independent on $\omega$, and $k_{z n}^{\mathrm{II}}=\bar{\omega}_{n} / a_{s}^{\mathrm{II}}$, where $\bar{\omega}_{n}$ is the frequency of the eigenharmonic of the oscillations. The solution of Eq. (10) then has the form

$\operatorname{Re} \bar{\omega}_{n}=\frac{\pi}{2} \frac{a_{s}^{\mathrm{II}}}{\Delta} n, \quad \operatorname{Im} \bar{\omega}_{n}=\frac{a_{s}^{\mathrm{II}}}{2 \Delta} \ln \frac{|1-Z|}{1+Z}$,

where $n=1,2, \ldots$ is the eigenharmonic number. The solution of Eq. (11) exists when $Z<1$, only for even harmonics of the eigen-oscillations $(n=2 k, k=1,2, \ldots)$, and when $Z>1$, only for odd harmonics $(n=2 k-1$, $k=1,2, \ldots)$. It is evident from Eq. (11) that $\operatorname{Im} \bar{\omega}_{n} \leq 0$ for all harmonics of the eigen-oscillations. This means that the eigenmodes are attenuated. Physically, this attenuation is caused by a partial penetration of the oscillations into the solar wind and into the magnetosphere. Of course, this process can also be treated from a different standpoint. Since 
the boundaries of the boundary layer are partially permeable, the eigen-oscillations inside the layer can be excited by magnetosonic waves incident on it from the outside. Of special interest is the analysis of the behaviour of the function $k_{z}^{2}\left(\boldsymbol{k}_{t}, \bar{\omega}^{(i)}\right)$ when calculating the wave energy flux from the solar wind to the magnetosphere. The presence of "transparency windows" in the domains of definition of $\omega$ and $\boldsymbol{k}_{t}$ has a substantial influence upon the magnitude of integral energy flux.

When $\boldsymbol{k}_{t}$ increases, both the solar wind and the magnetosphere become opaque for the waves under consideration, and then $\operatorname{Im} \bar{\omega}_{n}=0$, i.e. the transition layer becomes an ideal waveguide. The opacity condition for regions I and III has the form $k_{z}^{\mathrm{I}^{2}}\left(\boldsymbol{k}_{t}, \bar{\omega}^{\mathrm{I}}\right)<0$ and $k_{z}^{\mathrm{III}}{ }^{2}\left(\boldsymbol{k}_{t}, \bar{\omega}^{\mathrm{III}}\right)<0$, respectively.

\section{The function $k_{z}^{2}\left(\mathbf{k}_{t}, \bar{\omega}^{(i)}\right)$}

The function $k_{z}^{2}\left(\mathbf{k}_{t}, \bar{\omega}^{(i)}\right)$ exhibits the simplest behaviour in a non-moving medium, the magnetosphere. Let the vector $\boldsymbol{k}_{t}$ be described in a polar coordinate system $\boldsymbol{k}_{t}=\left(k_{t}, \theta\right)$, where $k_{t}=\sqrt{k_{x}^{2}+k_{y}^{2}}$ is the modulus of the tangential wave vector, and $\theta$ is the angle counted off from the axis $k_{x}$ in the same manner as shown in Fig. 1. Equation (6) may then be represented as

$k_{z}^{2}=-k_{t}^{2}+\frac{\bar{\omega}^{4}}{\left(\bar{\omega}^{2}-k_{t}^{2} c_{s}^{2} \cos ^{2} \theta\right) a_{s}^{2}}$,

where, for simplicity sake, the superscripts designating the layer parameters, are omitted. It is apparent from Eq. (12) that $\left|k_{z}^{2}\right|=\infty$ when $k_{t}=\left|\omega / c_{s} \cos \theta\right|$. Furthermore, at a fixed $\theta$ there exist two points

$\tilde{k}_{t}^{(1,2)}=\frac{\omega}{\sqrt{2} c_{s}|\cos \theta|}\left[1 \pm \sqrt{1-4 \frac{c_{s}^{2}}{a_{s}^{2}} \cos ^{2} \theta}\right]^{1 / 2}$,

where $k_{z}^{2}=0$. The "-" sign here corresponds to the superscript "(1)" of $\tilde{k}_{t}^{(1,2)}$, and the "+" sign corresponds to the index "(2)". When $k_{t}=0$ we have $k_{z}^{2}=\omega^{2} / a_{s}^{2}$, and when $k_{t} \rightarrow \infty$ we have $k_{z}^{2}=-k_{t}^{2}$. Figure 2a presents the general form of the function $k_{z}^{2}$ depending on $k_{t}$ at fixed $\omega$ and $\theta$. One can see that two intervals exist for magnetosonic waves, $0 \leq k_{t} \leq \tilde{k}_{t}^{(1)}$ and $\tilde{k}_{t}^{(2)} \leq k_{t} \leq\left|\omega / c_{s} \cos \theta\right|$ for which $k_{z}^{2}>0$. The first of them is for fast magnetosonic waves, and the second is for slow magnetosonic waves. For characteristic values of magnetospheric parameters, $a_{s} \gg c_{s}$, and the width of the first interval $\Delta k_{t}^{(1)} \sim \omega / a_{s}$ is much larger than the second one, $\Delta k_{t}^{(2)} \sim\left|\omega c_{s} \cos \theta / a_{s}^{2}\right|$.

In a medium in motion, the behaviour of $k_{z}^{2}$ is more complicated. Equation (12) in this case has two singular points

$\bar{k}_{t}^{(1,2)}=\frac{\omega}{\left(v^{(i)} \pm c_{s}\right)|\cos \theta|}$,

where $\left|k_{z}^{2}\right|=\infty$. The superscript "(1)" of $\bar{k}_{t}^{(1,2)}$ corresponds to the " + " sign, and the index "(2)" corresponds to
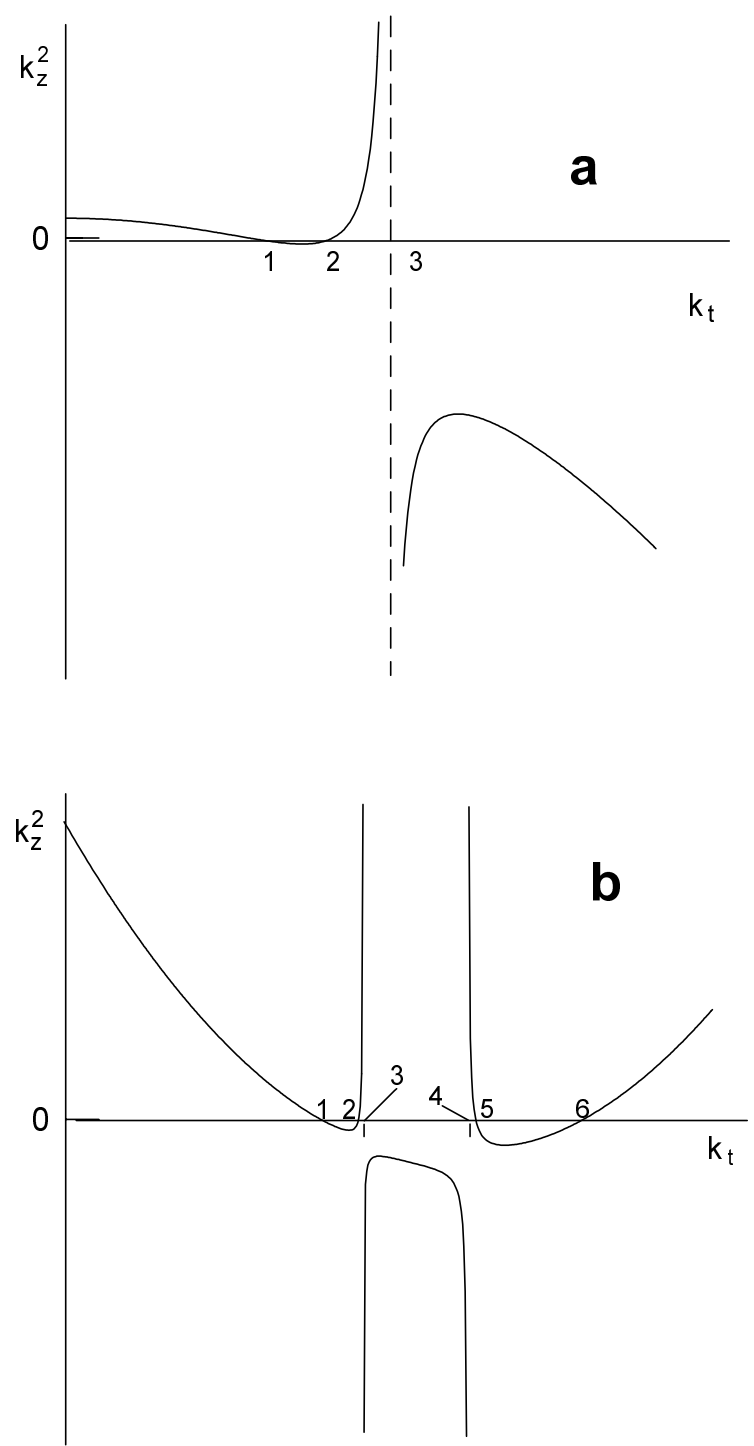

Fig. 2. The upper plot (a) presents the behaviour of $k_{z}^{2}$ in the magnetosphere at any values of $\theta$ and $\omega$. The points are numbered: $1-$ $k_{t}=\tilde{k}_{t}^{(1)}, 2-k_{t}=\tilde{k}_{t}^{(2)}, 3-k_{t}=\left|\omega / c_{s}^{(3)} \cos \theta\right|$. In a medium in motion, this plot corresponds to the sector $\theta^{*} \leq \theta \leq \pi-\theta^{*}$, and the points are numbered: $1-k_{t}=k_{t}^{(1)}, 2-k_{t}=k_{t}^{(3)}, 3-k_{t}=\bar{k}_{t}^{(1)}$. The lower plot (b) presents the behaviour of $k_{z}^{2}$ in a medium in motion in the sector $0 \leq \theta \leq \theta^{*}$. The points are numbered: $1-$ $k_{t}=k_{t}^{(1)}, 2-k_{t}=k_{t}^{(3)}, 3-k_{t}=\bar{k}_{t}^{(1)}, 4-k_{t}=\bar{k}_{t}^{(2)}, 5-k_{t}=k_{t}^{(4)}$, $6-k_{t}=k_{t}^{(2)}$.

the "-" sign in the denominator of this expression. In addition, Eq. (12) has four points

$k_{t}^{(m)}=\frac{\omega \tilde{k}_{t}^{(1,2)}}{\tilde{k}_{t}^{(1,2)} v^{(i)} \cos \theta \pm \omega}, \quad m=1,2,3,4$,

where $k_{z}^{2}=0$. The roots in Eq. (14) are numbered as follows. The superscript $m=1$ corresponds to $\tilde{k}_{t}^{(2)}$ in the numerator and to the " + " sign in the denominator of Eq. (14), $m=2$ to $\tilde{k}_{t}^{(2)}$ in the numerator and to the "-" sign in the denominator, 
$m=3$ to $\tilde{k}_{t}^{(1)}$ and to "+", and $m=4$ corresponds to $\tilde{k}_{t}^{(1)}$ and to "-". Here, $\tilde{k}_{t}^{(1,2)}$ is defined by the expression analogous to Eq. (13), with the difference that $a_{s}$ and $c_{s}$ correspond to parameters of the medium under consideration (the solar wind and the transition layer).

When $k_{t}=0$ we have $k_{z}^{2}=\omega^{2} / a_{s}^{2}$, and when $k_{t} \rightarrow \infty$

$k_{z}^{2} \cong k_{t}^{2} \frac{c_{s}^{2}-v^{2}\left(1-v^{2} \cos ^{2} \theta / a_{s}^{2}\right)}{v^{2}-c_{s}^{2}}$.

The behaviour of $k_{z}^{2}$ when $k_{t} \rightarrow \infty$ depends on the direction $\boldsymbol{k}_{t}$, i.e. on the value of the angle $\theta$. When $|\cos \theta|>$ $\left|\cos \theta^{*}\right| \equiv\left(a_{s}^{(i)} / v^{(i)}\right) \sqrt{1-\left(c_{s}^{(i)} / v^{(i)}\right)^{2}}$ we have $k_{z}^{2}>0$ (assuming $\left.c_{s}^{(i)}<v^{(i)}\right)$, and $k_{z}^{2}<0$ when $|\cos \theta|<\left|\cos \theta^{*}\right|$. The general form of the function $k_{z}^{2}$ depending on $k_{t}$ when $\theta<\theta^{*}$ is presented in Fig. 2b. There are two intervals for fast magnetosonic waves $0 \leq k_{t} \leq k_{t}^{(1)}$ and $k_{t}^{(2)} \leq k_{t}$, and two intervals for slow magnetosonic waves $k_{t}^{(3)} \leq k_{t} \leq \bar{k}_{t}^{(1)}$ and $\bar{k}_{t}^{(2)} \leq k_{t} \leq k_{t}^{(4)}$ for which $k_{z}^{2}>0$.

When $\theta^{*} \leq \theta \leq \pi-\theta^{*}$ the second intervals for fast and slow magnetosonic waves disappear, and the general form of the function $k_{z}^{2}$ is qualitatively of the same form as in Fig. 2a. In this case the interval for fast magnetosonic waves lies in the range $0 \leq k_{t} \leq k_{t}^{(1)}$, and for slow magnetosonic waves it lies in the range $k_{t}^{(3)} \leq k_{t} \leq \bar{k}_{t}^{(1)}$. As in the magnetosphere, the width of the intervals for fast magnetosonic waves is much larger compared with slow magnetosonic waves. If $\pi \geq \theta \geq \pi-\theta^{*}$, then $k_{z}^{2}>0$ for any $k_{t} \geq 0$ and the interval for slow magnetosonic waves disappears completely, and fast magnetosonic waves can propagate in the entire range $0 \leq k_{t}<\infty$.

One further remark refers to the stability of the concerned oscillations on the separation boundaries of the layers. If the oscillation is unstable, its frequency is a complex one $\omega=\omega_{r}+i \omega_{i}, \omega_{i}>0$. It is easy to check that for the oscillations with real values of $k_{t}^{2}>0$ considered in this study, the solution of Eq. (2) has complex values of $\omega$ only for waves for which $k_{z}^{2}<k_{t}^{2}\left(4 c_{s}^{2} \cos \theta / a_{s}^{2}-1\right)$. When $a_{s}^{2} \gg c_{s}^{2}$, which is assumed valid in the solar wind region, this leads to the condition $k_{z}^{2}<0$. In this paper we consider only the waves that propagate freely in the solar wind along the axis $z$. For such waves $k_{z}^{2}>0$ and, hence, they are stable and are not enhanced on the separation boundaries of the layers as they propagate from the solar wind to the magnetosphere.

\section{Magnetosonic wave energy flux and model of the spec- tral function}

The energy flux density is a quadratic function of the wave amplitude. For its normal component, we use the following expression

$$
\tilde{f}_{z}=\rho \frac{\omega\left(\bar{\omega}^{2}-k_{x}^{2} A^{2}\right)}{\bar{\omega}^{2} k_{z}}\left|\tilde{v}_{z}\right|^{2}
$$

obtained in a paper by McKenzie (1970), where it was shown that when the wave is incident on the sharp boundary, the law of conservation of total energy flux holds. In Appendix A, it is shown that the same law of conservation of total energy flux is also true for the problem of the wave incident on the transition layer, i.e. the equality holds:

$\tilde{f}_{z 3}=\tilde{f}_{z 1}+\tilde{f}_{z 2}$,

where subscripts 1, 2, 3 number the incident reflected and transmitted waves, in accordance with Fig. 1.

For an integration over the spectrum, let $\tilde{f}_{z}$ be expressed in terms of the disturbed density amplitude related to the disturbed velocity amplitude by the following relationship (Anderson, 1963)

$\tilde{v}_{z}=\frac{\bar{\omega}^{3}}{k^{2}} \frac{k_{z}}{\bar{\omega}^{2}-k_{x}^{2} A^{2}} \frac{\tilde{\rho}}{\rho}$.

From Eq. (15) we then have

$\tilde{f}_{z}=\frac{\omega \bar{\omega}^{4}}{k^{4}} \frac{k_{z}}{\bar{\omega}^{2}-k_{x}^{2} A^{2}} \frac{|\tilde{\rho}|^{2}}{\rho}$.

At the finite value of $\tilde{\rho}^{2}$ the expression (17) has no singularities for magnetosonic waves for which $k_{z}^{2}>0$.

Based on the fact that the width of the intervals of $k_{t}$ for fast magnetosonic waves with $k_{z}^{2}>0$ is much larger compared with those of slow magnetosonic waves, the contribution of slow magnetosonic oscillations will be neglected in what follows when calculating the energy flux density.

The characteristic form of the function $\tilde{f}_{z j}(j=1,2,3)$ when $\theta=0$ versus $k_{t}$ is plotted in Fig. 3. These plots are calculated on the basis of a model of the medium presented in Section 6 of this paper. In this case we have two intervals of $k_{t}$ for fast magnetosonic waves with $k_{z}^{2}>0$ in the solar wind and one interval in the magnetosphere. The relationship between the incident wave amplitude and the amplitude of the transmitted and reflected waves is given by the expressions (7), (8), (9) and (16). Two cases are considered. One refers to the wave incident on the solar wind-magnetosphere interface in the absence of the transition layer $(\Delta=0)$. One can see that in the "transparency window" of the magnetosphere about $70 \%$ of the incident wave energy flux penetrates to its interior. The second case refers to the wave incident on the transition layer $\Delta=10^{4} \mathrm{~km}$ in thickness. Energy flux densities of the transmitted and reflected waves become modulated in accordance with the modulation of the coefficients $R$ and $T$. In the second interval of $k_{t}$ for fast magnetosonic waves with $k_{z}^{2}>0$ in the solar wind, the modulation is lacking, because at given values of $\omega$ and $\theta$ the magnetosphere turns out to be opaque for the waves under consideration, and they are totally reflected. Energy flux maxima of the transmitted and reflected waves in this case coincide with the magnitude of these fluxes in the absence of the transition layer.

The expression (17) is written for the energy flux density of the wave with a given frequency $\omega$ and a wave vector $\boldsymbol{k}_{t}$. To determine the total energy density of the waves, it is necessary to perform an inverse Fourier transform of Eq. (17) over the entire spectrum of frequencies and wave vectors of 


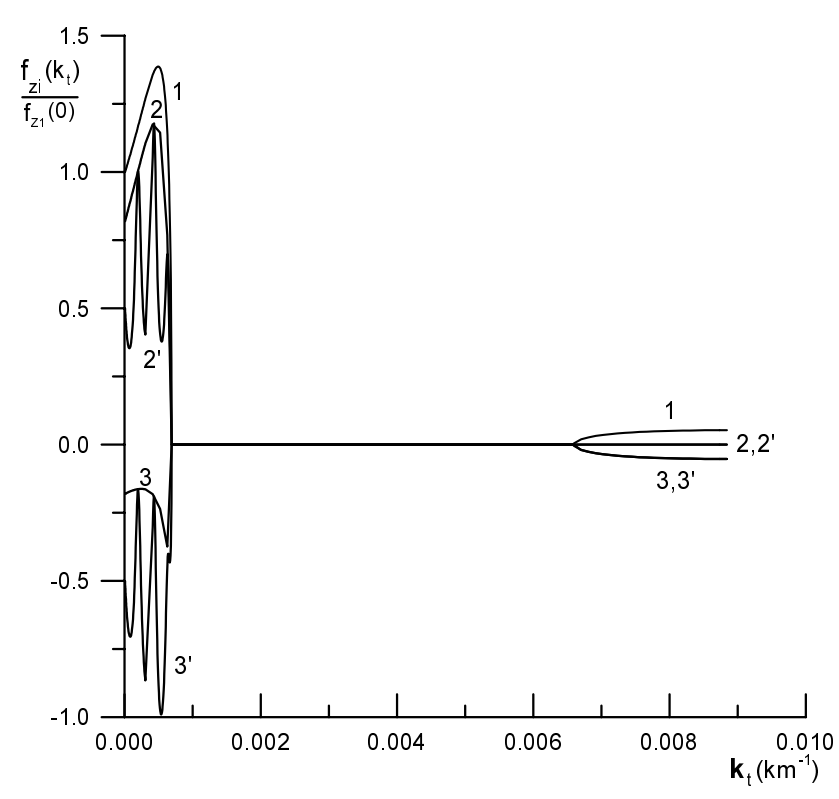

Fig. 3. Typical appearance of the energy flux densities of a monochromatic magnetosonic wave in the solar wind and in the magnetosphere in the sector $0 \leq \theta \leq \theta^{*}$ depending on $k_{t}$. The energy flux densities are numbered: 1 - incident wave; 2 - wave transmitted to the magnetosphere at $\Delta=0$ and $2^{\prime}-$ at $\Delta=10^{4}$ $\mathrm{km} ; 3$ - wave reflected from the transition layer at $\Delta=0$ and $3^{\prime}-$ at $\Delta=10^{4} \mathrm{~km}$.

the oscillations under consideration. Suppose that the oscillation spectrum is symmetric with respect to a change in the sign of $\omega$. The inverse Fourier transform then has the form

$f_{z}=\frac{2}{(2 \pi)^{3 / 2}} \int_{0}^{\infty} \mathrm{d} \omega \int_{0}^{\pi} \mathrm{d} \theta \int_{0}^{\infty} \tilde{f}_{z}\left(k_{t}, \theta, \omega\right) k_{t} \mathrm{~d} k_{t}$.

As follows from Eq. (17), at a fixed value of $\tilde{\rho}^{2}$ the integral of Eq. (18) turns out to be divergent. To avoid this, it is necessary to specify the spectrum $\tilde{\rho}^{2}$ to ensure the convergence of this integral.

Here we shall use the model spectrum function constructed by Leonovich and Mishin (1999). This function features well the properties of a spectrum of magnetosonic waves observed in the solar wind (Matthaeus and Goldstein , 1982; Goldstein et al., 1995; Marsch and Tu, 1990). These spectra were measured in the solar wind region ahead of the bow shock. It will be assumed, however, that the spectrum of turbulent oscillations in the tail part of the magnetosphere considered here has a structure similar to that used in the cited references. In other words, it will be assumed that the oscillation spectrum is carried by the solar wind stream to the magnetospheric region under consideration without substantially modifying their spectrum.

The expression of the Fourier harmonic of the mean square of amplitude of density oscillations is set by Leonovich and Mishin, (1999)

$\tilde{\rho}^{2}=C \omega^{-\alpha} k_{t}^{-2 \beta}$
Table 1.

\begin{tabular}{ccccc}
\hline $\begin{array}{c}\text { region } \\
(i)\end{array}$ & $\begin{array}{c}v^{(i)} \\
(\mathrm{km} / \mathrm{s})\end{array}$ & $\begin{array}{c}B^{(i)} \\
(\mathrm{nT})\end{array}$ & $\begin{array}{c}n^{(i)} \\
\left(\mathrm{cm}^{-3}\right)\end{array}$ & $\begin{array}{c}T^{(i)} \\
\left(10^{5} \mathrm{~K}\right)\end{array}$ \\
\hline I & 400 & 5 & 5 & 5.75 \\
\hline II & 200 & 7.5 & 3 & 6.6 \\
\hline III & 0 & 10 & 1 & 7.2 \\
\hline
\end{tabular}

where $C$ is a certain amplitude that can be determined by performing an inverse Fourier transform over the entire spectrum of frequencies and wave vectors. The spectral function (19) ensures the convergence of the integral (18) when $k_{t} \rightarrow \infty$; however, the integral becomes divergent when $k_{t}=0$. Hence the spectrum (19) should be cut off when $k_{t} \rightarrow 0$. It is known from a paper by Matthaeus and Goldstein (1982) that there exists a maximum correlation scale $\hat{l}$ associated with the inhomogeneous structure of the solar wind. For magnetic field oscillations, it is $\hat{l}=1.12 \times 10^{6}$ $\mathrm{km}$. This scale may be thought of as being maximal for magnetosonic oscillations, and the oscillation spectrum (19) must then be bounded below by a minimum value of $\hat{k}_{t}=2 \pi / \hat{l}$. Correspondingly, it is also possible to introduce a minimum oscillation frequency $\hat{\omega}=\hat{k}_{t} v^{(1)}$ bounding the oscillation frequency spectrum below.

Allowance must also be made for the presence in the oscillation spectrum of "transparency windows" taken up in Sect. 4. The amplitude $C$ in the spectral function (19) meeting these requirements has the form

$C=<\rho_{1}^{2}>\Phi\left(\mathbf{k}_{t}, \omega\right) \tilde{C}$,

where $<\rho_{1}^{2}>$ is the mean square of the density oscillation amplitude of the waves incident on the transition layer, and $\Phi\left(\boldsymbol{k}_{t}, \omega\right)$ is the filter that takes into account the typical cutoff scale of the spectrum and the presence of "transparency windows" (see Leonovich and Mishin, 1999). The normalizing factor is chosen such that

$$
\frac{2 \tilde{C}}{(2 \pi)^{3 / 2}} \int_{0}^{\infty} \omega^{-\alpha} \mathrm{d} \omega \int_{0}^{\infty} \Phi\left(\mathbf{k}_{t}, \omega\right) k_{t}^{-2 \beta+1} \mathrm{~d} k_{t}=1 .
$$

\section{Results of numerical calculation and discussion}

We now carry out numerically an inverse Fourier transform Eq. (18) using for $\tilde{\rho}^{2}$ the model expression (19) with the amplitude (20). To accomplish this, it is necessary to specify parameters of the medium. For the magnetosheath (region I), we employ parameters averaged over many observations. For the transition layer (region II) and the magnetosphere (region III), we pick parameters balanced out with solar wind parameters according to condition (1). The above parameters are presented in the Table 1. 
It is necessary to specify, in addition to equilibrium parameters, the mean amplitude of density oscillations associated with magnetosonic waves in the solar wind, $\left\langle\left|\rho_{1}\right|>\right.$. According to observations, the density oscillation amplitude of solar wind plasma makes up 20\%-50\% (Matthaeus and Goldstein, 1982; Goldstein et al., 1995) of its mean value. For the mean oscillation amplitude, we choose the value $<\left|n_{1}\right|>=1 \mathrm{~cm}^{-3}$, which makes up $20 \%$ of the mean density $n^{\mathrm{I}}=5 \mathrm{~cm}^{-3}$. The mean value of the oscillation density amplitude is defined as $<\left|\rho_{1}\right|>=<\left|n_{1}\right|>m_{p}$, where $m_{p}$ is the mass of a proton.

Our chosen model neglects the finite size of the magnetosphere. To take this into account, we define the "geoeffective" oscillation energy flux such that, when taking the integral (18) around the variable $k_{t}$ in the magnetosphere, we will use the quantity $\hat{k}_{t}=2 \pi / l$ as the lower limit, where $l$ is the typical largest size of the magnetospheric tail. It is known that the value of $l$ varies through a significant range. According to Cowley (1992) and Mishin et al. (2000), early in the substorm growth phase the effective tail length is $l \approx 30 R_{E}$ (where $R_{E}$ is the Earth's radius). Within the course of the substorm growth phase the tail length increases and reaches, by the time of breakup, a value close to the correlation scale of solar wind inhomogeneities, $l \sim \hat{l} \approx 10^{6} \mathrm{~km}$. Thus, while at the beginning of the growth phase the "geoeffective" energy flux of the waves constitutes only a portion of the total flux capable of penetrating the magnetosphere's interior, at the end of this phase the entire flux penetrating the magnetosphere becomes "geoeffective".

"Geoeffective" energy flux densities are plotted in Fig. 4 (three curves at the bottom, and the vertical axis $f_{z 3}$ at the left). Flux densities are given for four different values of the transition layer thickness, $\Delta=0,10^{4}, 2 \times 10^{4} \mathrm{~km}$. For comparison, the figure shows the energy density flux of the waves incident on the transition layer from the solar wind (the upper horizontal line $f_{z}=9.8 \mathrm{~W} / \mathrm{km}^{2}$ ). One can see that at the beginning of the growth phase $\left(l \approx 30 R_{E}\right)$ the "geoeffective" energy flux density makes up $1-2 \%$ of the energy flux density of the incident waves. By the end of this phase it reaches values from $20 \%$ (for $\Delta=2 \times 10^{4} \mathrm{~km}$ ) to $40 \%$ (for $\Delta=0$ ) of the flux density of the incident waves. Hence the presence of the transition layer does not drastically affect the value of the wave energy flux to the magnetosphere. As the layer thickness varies from $\Delta=0$ to $\Delta=2 \times 10^{4} \mathrm{~km}$, the flux $f_{z 3}$ decreases by a factor of 2 . So, the main results obtained by Leonovich and Mishin (1999) are not drastically changed. This is because the main contribution to the energy flux is made by the longest-wave oscillation harmonics, for which $k_{z}^{\mathrm{II}} \Delta \ll 1$, i.e. the transition layer for them is thin.

Hence, it follows that our obtained results differ essentially from those reported by Verzariu (1973) and Wolfe and Kaufmann (1975), where the energy flux of magnetosonic waves penetrating deep into the magnetosphere is estimated at a level of $1-2 \%$ of the flux incident on the magnetosphere from the solar wind. Specifically, such a difference is not caused by taking into account the transition layer with two sharp boundaries - the presence of the transition layer reduces the

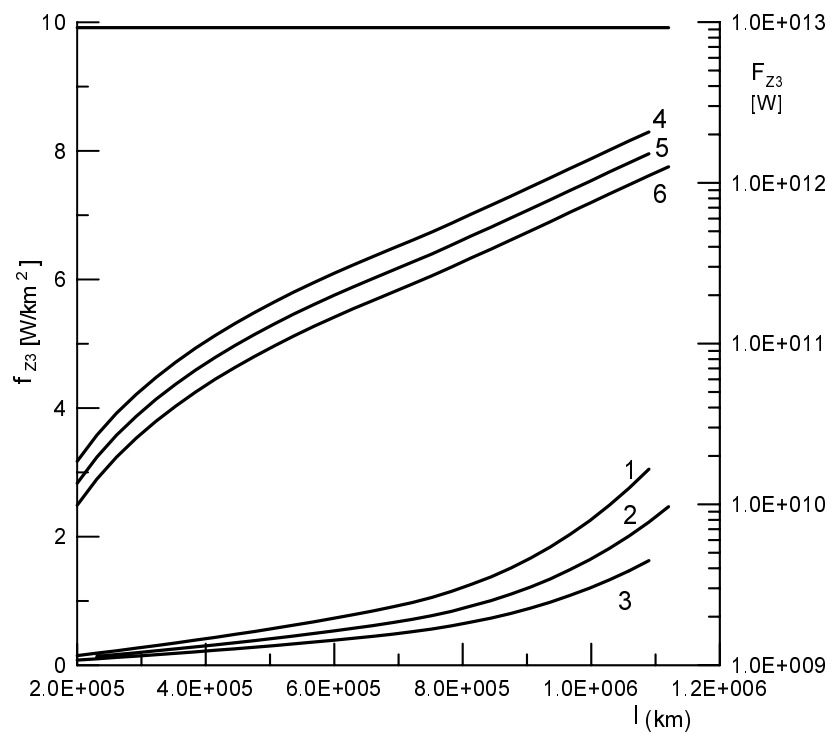

Fig. 4. Dependence of the total "geoeffective" energy flux density of the magnetosonic waves penetrating into the magnetosphere (curves 1-3 and the vertical axis $f_{z 3}$ at the left) on the magnetospheric tail length $-l$. The numbered curves correspond to the transition layer thickness: $1-\Delta=0 ; 2-\Delta=10^{4} \mathrm{~km} ; 3-\Delta=2 \times 10^{4}$ $\mathrm{km}$. The energy density level of the waves incident on the transition layer $f_{z 3} \simeq 9.8 \mathrm{~W} / \mathrm{km}^{2}$ is shown at the top.

Curves 4-6 (and the vertical axis $F_{z 3}$ at the right) represent the integral (throughout the entire lateral surface of the magnetosphere) "geoeffective" wave energy flux for the same values of the thickness $\Delta$ as in plots $1-3$ (correspondence $4 \longleftrightarrow 1,5 \longleftrightarrow 2,6 \longleftrightarrow 3$ ).

magnetosonic wave flux penetrating into the magnetosphere. We anticipate that this difference is due to two factors.

The first factor implies that the energy flux of magnetosonic waves from the solar wind to the magnetosphere was estimated by Verzariu (1973) and Wolfe and Kaufmann (1975) for the subsonic regime of flow around the magnetosphere. This is quite true for the dayside part of the magnetopause that was treated in the above references. In this paper, regarding the tail region of the magnetosphere, we consider the plasma stream propagating with respect to the magnetosphere with a supersonic speed. This difference leads to the fact that the relative value of the "transparency window" in the case of a supersonic stream is several times larger when compared with the subsonic stream. Accordingly, the part of the wave energy flux that propagated into the magnetosphere increases proportionally with it. According to McKenzie (1970), this difference at the transition from the subsonic regime of flow to the supersonic regime corresponds to an increase in the sector of incidence angles of the waves having a relatively large transmission coefficient (see Figs. $4 \mathrm{a}$ and $\mathrm{b}$ in the cited reference).

The second factor implies that Verzariu (1973) and Wolfe and Kaufmann (1975) assumed that the energy flux of the magnetosonic waves incident on the magnetosphere is uniformly distributed in all incidence angles. However, as is evident from Sect. 4 of this paper, with a given wave fre- 
quency $\omega$, in the solar wind there is a range of values of $\bar{k}_{t}^{(1)}<k_{t}<\bar{k}_{t}^{(2)}$ at which magnetosonic waves cannot propagate along the axis $z$. The flow of monochromatic waves consists of two parts: waves with $k_{t} \leq k_{t}^{(1)}$, and waves with $k_{t} \geq k_{t}^{(2)}$. As follows from the reasoning presented in Sect. 5, most of the energy flux of magnetosonic oscillations of the solar wind is concentrated in the range of small values of $k_{t}$. It is for such oscillations that there is a "transparency window" $k_{t} \leq \tilde{k}_{t}^{(1)}$, leading to the fact that up to $40 \%$ of the wave energy flux penetrate from the solar wind into the magnetosphere.

Figure 4 also plots the total energy flux penetrating the magnetosphere through its lateral surface (the upper four curves and the vertical axis $F_{z 3}$ at the right). The magnetosphere in this case is represented as a cylinder of radius $R=16 R_{E}$ and length $l$. In the early stage of the substorm growth phase the "geoeffective" energy flux is (12 ) $\times 10^{10} \mathrm{~J} / \mathrm{s}$ and reaches values from $1.5 \times 10^{12} \mathrm{~J} / \mathrm{s}$ (for $\Delta=2 \times 10^{4}$ ) to $3 \times 10^{12} \mathrm{~J} / \mathrm{s}$ (for $\Delta=0$ ) at the end of this phase. This energy flux is quite sufficient for sustaining a stationary magnetospheric convection (according to estimates made by Axford (1964), the required mean energy flux is $10^{11} \mathrm{~J} / \mathrm{s}$ ).

On the basis of the above calculations it is possible to estimate the total energy input to the magnetosphere with the "geoeffective" flux during a time interval of the order of the time of the substorm growth phase, $\Delta t \approx 3 \times 10^{3} \mathrm{~s}$. In order for such an estimate to be made, it is necessary to specify the law of variation of the tail length from $l=l_{\min } \approx 30 R_{E}$ to $l=l_{\max }$, where the correlation scale of the solar wind inhomogeneities $\hat{l}$ may be used as $l_{\max }$. We take the following model for the tail length dynamics

$l=l_{\min }+\left(\frac{t}{\Delta t}\right)^{\gamma} \Delta l$,

where $\Delta l=l_{\max }-l_{\min }$. The total energy input to the magnetosphere for the time $\Delta t$ with the "geoeffective" flux is

$$
\begin{gathered}
W=2 \pi R \int_{0}^{\Delta t} F_{z 3}(l(t)) l(t) \mathrm{d} t= \\
2 \pi R \int_{l_{\min }}^{l_{\max }} F_{z 3}(l)\left(\frac{\mathrm{d} l}{\mathrm{~d} t}\right)^{-1} l \mathrm{~d} l .
\end{gathered}
$$

From (21) we have

$$
\frac{\mathrm{d} l}{\mathrm{~d} t}=\gamma\left(\frac{l-l_{\min }}{\Delta l}\right)^{(\gamma-1) / \gamma} \frac{\Delta l}{\Delta t} .
$$

A substantial role in our estimate is played by the index of intense growth of the tail, $\gamma$. As shown by Mishin et al. (1996), different scenarios for growth of the effective tail length, both slow $(\gamma<1)$ and fast $(\gamma>1)$, can be realized in different substorm events. In this connection, we have constructed the dependences of $W(\gamma)$ in the range $0.5 \leq \gamma \leq 5$, Fig. 5 .

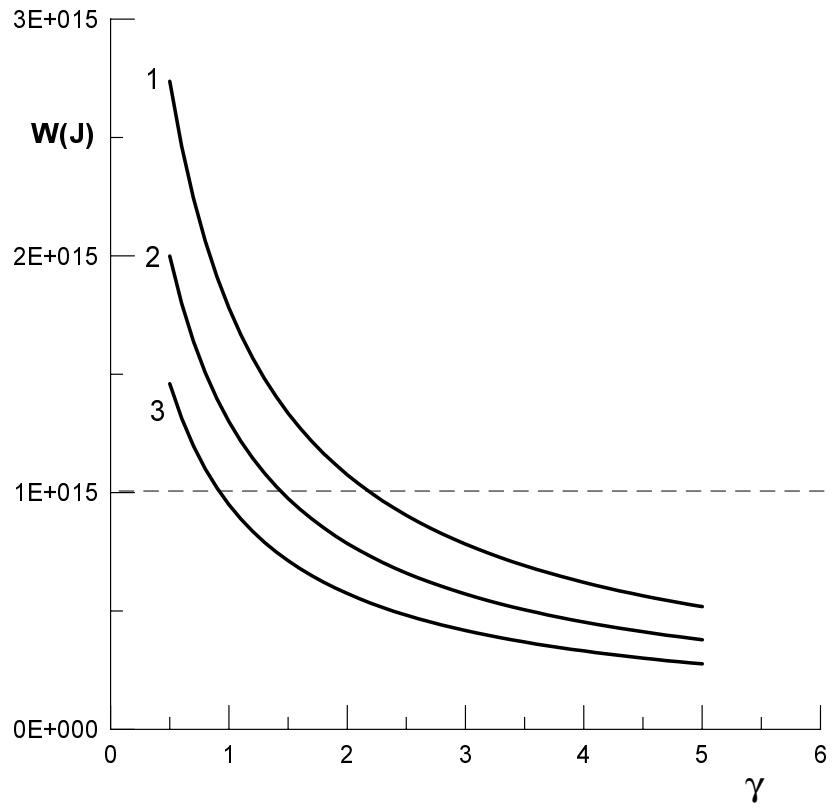

Fig. 5. Dependence of the total energy $W$ supplied into the magnetosphere with the "geoeffective" energy flux during the time $\Delta t=3 \times 10^{3} \mathrm{~s}$ on the growth intensity index of the tail $\gamma$ in the model Eq. (21). Curve numbers correspond to different thicknesses of the transition layer: $1-\Delta=0,2-\Delta=10^{4} \mathrm{~km}, 3-$ $\Delta=2 \times 10^{4} \mathrm{~km}$.

With an increase in $\gamma$, the total energy input to the magnetosphere decreases. The reason is that a maximum wave energy flux enters into the magnetosphere when the magnetospheric tail length is at its maximum. With a slow growth rate of the tail $(\gamma<1)$, the magnetosphere remains in a state with maximum tail length during a long time interval, and hence, during $\Delta t$ it receives more energy than in the case of a fast tail growth $(\gamma>1)$. For comparison, this figure also shows the level of energy released during a moderate substorm $\left(W \sim 10^{15} \mathrm{~J}\right)$. It is evident that in some scenarios for growth of the magnetospheric tail, the "geoeffective" energy input to the magnetosphere exceeds this level.

The dayside magnetosphere, disregarded in our paper, can make an additional contribution to the total wave energy flux comparable with the energy flux through the lateral surface.

\section{Conclusions}

We now summarize the main results of this study:

1. We have determined the transmission and reflection coefficients of magnetosonic waves in solving the problem of their transmission from the solar wind to the magnetosphere through the transition layer.

2. It has been shown that eigenmodes of magnetosonic oscillations exist in the transition layer, and they attenuate due to the escape of a portion of their energy to the solar wind and the magnetosphere. 
3. Using the model expression for the spectrum of solar wind oscillations we have carried out calculations of the "geoeffective" energy flux penetrating the magnetosphere. It has been shown that the magnitude of this flux is sufficient for sustaining a stationary magnetospheric convection.

4. An estimate was made of the total energy transferred to the magnetosphere by the "geoeffective" flux of magnetosonic waves during a time interval of the order of the time of the substorm growth phase. It has been shown that this energy is quite comparable with the moderate substorm energetics.

\section{Appendix A Conservation of the energy flux}

We use for $\tilde{f}_{z 2}$ and $\tilde{f}_{z 3}$ the expression (15), in which $\tilde{v}_{z 2}^{2}=$ $|R|^{2} \tilde{v}_{z 1}^{2}, k_{z 2}^{\mathrm{I}}=-k_{z 1}^{\mathrm{I}}$ for $\tilde{f}_{z 2}$ and $\tilde{v}_{z 3}^{2}=|T|^{2} \tilde{v}_{z 1}^{2}$ for $\tilde{f}_{z 3}$, where $R$ and $T$ are defined by the expressions (8) and (9). We have

$$
\begin{aligned}
\tilde{f}_{z 3}-\tilde{f}_{z 2}= & \omega\left[\frac{\rho^{\mathrm{III}}}{k_{z}^{\mathrm{III}}} \frac{\bar{\omega}^{\mathrm{III}}-k_{x}^{2} A^{\mathrm{III}}}{\bar{\omega}^{2}}|T|^{2}+\right. \\
& \left.\frac{\rho^{I}}{k_{z}^{I}} \frac{\bar{\omega}^{\mathrm{I}^{2}}-k_{x}^{2} A^{\mathrm{I}^{2}}}{\bar{\omega}^{\mathrm{I}^{2}}}|R|^{2}\right] \tilde{v}_{z 1}^{2} .
\end{aligned}
$$

It can be shown that

$|R|^{2}=1-\frac{\bar{\omega}^{\mathrm{I}^{2}}}{\bar{\omega}^{\mathrm{III}}} Z_{1} Z_{2}|T|^{2}$.

Then

$\tilde{f}_{z 3}-\tilde{f}_{z 2}=\omega \frac{\rho^{\mathrm{I}}}{k_{z}^{\mathrm{I}}} \frac{\bar{\omega}^{\mathrm{I}^{2}}-k_{x}^{2} A^{\mathrm{I}^{2}}}{\bar{\omega}^{\mathrm{I}^{2}}} \tilde{v}_{z 1}^{2}=\tilde{f}_{z 1}$,

i.e. conservation of the incident wave energy flux occurs.

Acknowledgements. We are grateful to V.G. Mikhalkovsky for his assistance in preparing the English version of the manuscript.

This work is partially supported by the grants of Russian Foundation for Basic Research (RFBR No 02-05-39020) and National Scientific Foundation of China (NSFC No 40025413).

Topical Editor G. Chanteur thanks J. de Keyser and another referee for their help in evaluating this paper.

\section{References}

Akasofu S.-I., Hones, Jr., E. W., Bame, S. J., Asbridge, J. R., and Lui, A. T: Magnetotail and boundary layer plasmas at a geocentric distance of $\sim 18 R_{E}$ : Vela 5 and 6 observation, J. Geophys. Res., 78, 7257-7274, 1973.

Anderson, J. E.: Magnetohydrodynamic shock waves, 297 pp., M.I.T. Press, Cambridge, 1963.

Axford, W. I.: Viscous interaction between the solar wind and the Earth's magnetosphere, Planet. Space Sci., 12, 45-53, 1964.

Belmont, G. and Rezeau, L.: Magnetopause reconnection induced by magnetosheath Hall-MHD fluctuations, J. Geophys. Res., 106, 10 751-10 760, 2001.
Brekhovskikh, L. M.: Waves in layered media, 323 pp., Academic Press, N.Y., 1980.

Cowley, S. W. H.: The role and location of magnetic reconnection in the geomagnetic tail during substorms, Proceedings of ICS-1, 401-404, ESA SP-335, 1992.

De Keyser, J., Roth, M., Reberac, F., Rezeau, L., and Belmont, G.: Resonant amplification of MHD waves in realistic subsolar magnetopause configurations, J. Geophys. Res., 104, 23992409, 1999.

De Keyser, J. and Cadez, V.: Excitation of low-frequency fluctuations at the magnetopause by intermittent broadband magnetosheath waves, J. Geophys. Res., 106, 29467-29477, 2001.

Farrugia, C. J., Gratton, F. T., Contin, J., Cocheci, C. C., Arnoldy, R. L., Ogilvie, K. W., Lepping, R. P., Zastenker, G. N., Nozdrachev, M. N., Fedoriv, A., Sauvad, J.-A., Steinberg, J. T., and Rostoker, G.: Coordinated Wind, Interball/tail, and ground observations of Kelvin-Helmholtz waves at the near-tail, equatorial magnetopuse at dusk: January 11, 1997, J. Geophys. Res., 105, 7639-7669, 2000.

Goldstein, M. L., Roberts, D. A., and Matthaeus, W. H.: Magnetohydrodynamic turbulence in the solar wind, Ann. Rev. Astron. Astrophys., 33, 283-325, 1995.

Greifinger, P.: Ionospheric propagation of oblique hydromagnetic plane waves at micropulsation frequencies, J. Geophys. Res., 77, 2377-2391, 1972.

Lee, L. C., Albano, R. K., and Kan, J. R.: Kelvin-Helmholtz instability in the magnetopause boundary layer region, J. Geophys. Res., 86, 54-58, 1981.

Leonovich, A. S. and Mazur, V. A.: An electromagnetic field, induced in the ionosphere and atmosphere by low-frequency Alfvén oscillations of the magnetosphere: general theory, Planet. Space Sci., 39, 529-546, 1991.

Leonovich, A. S. and Mishin, V. V.: An energy flux of magnetosonic waves from the solar wind into magnetosphere, (in Russian), Geomagnetizm i aeronomiya, 39, 52-58, 1999.

Leonovich, A. S.: A theory of field line resonance in a dipole-like axisymmetric magnetosphere, J. Geophys. Res., 106, 25803 $25812,2001 \mathrm{a}$.

Leonovich, A. S. and Mazur, V. A.: On the spectrum of magnetosonic eigenoscillations of an axisymmetric magnetosphere, J. Geophys. Res., 106, 3919-3928, 2001 b.

Mann, I. R., Wright, A. N., Mills, K. J., and Nakariakov, V. N.: Excitation of magnetospheric waveguide modes by magnetosheath flows, J. Geophys. Res., 104, 333-353, 1999.

Marsh, E. and Tu, C.-Y.: Spectral and spatial evolution of compressive turbulence in the inner solar wind, J. Geophys. Res., 95A, 11945-11 956, 1990.

Matthaeus, W. H. and Goldstein, M. L.: Measurement of the rugged invariants of magnetohydrodynamics turbulence in the solar wind, J. Geopys. Res., 87A, 6011-6028, 1982.

McKenzie, J. F.: Hydromagnetic wave interaction with the magnetopause and the bow shock, Planet. Space Sci., 18, 1-23, 1970.

Mishin, V. V.: About wave energy flow into the magnetosphere under action of the solar wind pressure oscillations, Issledovaniya po geomagnetizmu aeronomii i fizike Solntsa, 104, 182-185, 1996.

Mishin, V. M., Russell, C. T., Bazarzhapov, A. D., and Saifutdinova, T. I.: Study of weak substorms observed during December 8, 1990, Geospace Environment Modelling campaign: timing of different types of substorm onsets, J. Geophys. Res., 105, $23263-23276,2000$.

Rezeau, L. and Belmont, G.: Magnetic turbulence at the 
magnetopause, a key problem for understanding the solar wind/magnetosphere exchanges, Space Sci. Rev., 84, 427-441, 2001.

Sckopke, N. and Paschmann, G.: The plasma mantle. A survey of magnetotail boundary layer observations, J. Atmosph. Terr. Phys., 40, 261-278, 1978.

Tu, C.-Y., Roberts, D. A., and Goldstein, M. L.: Spectral evolution and cascade constant of solar wind Alfvénic turbulence, J. Geophys. Res., 94A, 13 575-13 586, 1989.
Verzariu, P.: Reflection and refraction of hydromagnetic waves at the magnetopause, Planet. Space Sci., 21, 2213-2225, 1973.

Wolfe, A. and Kaufmann, R. L.: MHD wave transmission and production near the magnetopause, J. Geophys. Res., 80, 17641775, 1975.

Yumoto K. and Saito, T.: Hydromagnetic waves driven by velocity shear instability in the magnetospheric boundary layer, Planet. Space Sci., 28, 789-798, 1980. 